\title{
用双色三光子共振光电离光谱法测量 铀原子奇宇称高激发态能级
}

\author{
金昌泰 李玉福 梁秀清 王成飞 \\ 安玉贤 王秀兰宋伯学 周大凡 \\ (中国科学院长春应用化学研究所, 长春 130022)
}

\section{关键词铀原子、高激发态、共振电离}

数以干计的铀原子能级正在发现之中, 但铀原子光谱复杂, 给测定工作带来很多困难. 绝 大多数铀原子能级信息是在常规光源中 (如空心阴极或无电极放电灯) 由发射或者吸收光谱分 析所获得 ${ }^{[1]}$, 其光谱数据大多数属于铀原子低能级和中间态能级, 高激发态极少. 近几年来利 用激光多步光电离或苂光测量技术, 获得了许多新的铀原子能级, 但公开发表的较少 ${ }^{[2,3]}$. 我 们以铀原子双色三光子共振光电离光谱法, 在 $32000-34000 \mathrm{~cm}^{-1}$ 范围内测得了 90 个铀原子 奇宇称高激发态能级并确定了能级的 $J$ 值.

\section{一、实 验}

本实验采用的实验装置, 类似于铀原子单色三光子电离光谱的实验装置 ${ }^{[4]}$. 不同之处, 在 于本实验利用 $\mathrm{Nd}$ : YAG 二倍频激光同步百浦的两台脉冲染料激光器 $\mathrm{DL}_{1}$ 和 $\mathrm{DL}_{2}$, 采用激光 染料 $\mathrm{R} 640$ 和 $\mathrm{R} 610$, 激光波长范围为 $578-623 \mathrm{~nm}$. $\mathrm{DL}_{1}$ 与 $\mathrm{DL}_{2}$ 激光在同轴相反方向上进 人作用区, 与原子束垂直相交. $\lambda_{1}$ 与铀原子第一步跃迁波长值共振. $\mathrm{DL}_{1}$ 固定在 $\lambda_{1}$ 上, 扫描 $\mathrm{DL}_{2}$. 监测光电离信号. $\mathrm{DL}_{1}$ 激光把铀原子从基态激发到 $16000-18000 \mathrm{~cm}^{-1}$ 范围内的能级, $\mathrm{DL}_{2}$ 激光, 进一步激发到 $32000-34000 \mathrm{~cm}^{-1}$ 范围内的奇宇称能级. 由于在 $\mathrm{DL}_{2}$ 中引人时间延 迟, 在作用区, $\mathrm{DL}_{2}$ 激光脉冲相对 $\mathrm{DL}_{1}$ 激光脉冲延迟 10ns, 这样确保时序吸收 $\mathrm{DL}_{1}$ 与 $\mathrm{DL}_{2}$ 的 光子, 铀原子最后从 $\mathrm{DL}_{2}$ 再吸收一个光子而电离. 铀离子由偏转电极 $(2000 \mathrm{~V} / \mathrm{cm})$ 偏转, 经飞 行时间质谱和 M162 Boxcar 积分器接收、放大, 送人 X-Y 双笔记录仪的一个通道. 为标定激 光波长, 采用 $\mathrm{Ne}$ 光电流峰作为波长标准. 光电流信号经单点门限积分器放大, 输人到同一台 $\mathrm{X}-\mathrm{Y}$ 双笔记录仪的另一个通道, 与电离信号同时记录. 由此法标定波长的精确度为 $0.01 \mathrm{~nm}$.

\section{二、结果与讨论}

在双色三光子电离谱图中, 除双色峰外还有单色峰. 必须将这两种共振电离峰加以区别. 为此, 挡掉 $\mathrm{DL}_{1}$, 扫描 $\mathrm{DL}_{2}$ 激光获得单色三光子电离谱图. 在双色三光子电离谱中首先挑选出 单色峰, 而后对剩下的每一个双色峰进一步逐个进行辨认. 图 1 给出 $\mathrm{DL}_{1}$ 固定在 $605.68 \mathrm{~nm}$

1992-01-20 收稿, 1992-04-18 收修改稿

第 19 期

科 学 通 报 
表 1 由两个以上中间态所获得的铀原子奇宇称高激发态能级

\begin{tabular}{|c|c|c|c|c|c|}
\hline & 级 & & & 献 & \\
\hline 能 $\left(\mathrm{cm}^{-1}\right)^{\text {级 }}$ & 被激发能级 & $J$ & 能 $\left(\mathrm{cm}^{-\mathbf{t}}\right)$ & $\boldsymbol{J}$ & 参考文献 \\
\hline 33018.67 & $A, C$ & 6 & & & \\
\hline 33055.55 & $A, C$ & 6 & & & \\
\hline 33096.54 & $A, B, C$ & 6 & 33096.35 & 6 & {$[2,3]$} \\
\hline 33118.76 & $A, B$ & 6,7 & 33119.00 & 6,7 & {$[2,3]$} \\
\hline 33129.29 & $A, B, C$ & 6 & 33129.26 & 6 & {$[2,3]$} \\
\hline 33192.44 & $A, B$ & 6,7 & & & \\
\hline 33212.51 & $\mathrm{~B}, \mathrm{C}$ & 5,6 & 33212.72 & 5 & {$[2,3]$} \\
\hline 33215.99 & $\mathrm{~B}, \mathrm{C}$ & 5,6 & & & \\
\hline 33251.79 & $A, B, C$ & 6 & 33252.42 & 6 & {$[2,3]$} \\
\hline 33263.52 & A, B & 6,7 & 33264.30 & 6,7 & {$[2,3]$} \\
\hline 33275.48 & $\mathrm{~B}, \mathrm{C}$ & 5,6 & 33275.16 & 5,6 & {$[2,3]$} \\
\hline 33323.07 & $\mathrm{~B}, \mathrm{C}$ & 5,6 & 33323.08 & 5,6 & {$[2,3]$} \\
\hline 33350.03 & $B, C$ & 5,6 & & & \\
\hline 33378.57 & $A, B, C$ & 6 & 33378.78 & 6 & {$[2,3]$} \\
\hline 33421.27 & $A, B, C$ & 6 & 33421.12 & 6 & [3] \\
\hline 33474.99 & $A, B$ & 6,7 & 33474.95 & 6 & {$[2,3]$} \\
\hline 33492.11 & $A, B$ & 6,7 & & & \\
\hline 33516.71 & $A, B$ & 6,7 & 33516.86 & 6 & {$[2,3]$} \\
\hline 33549.76 & $\mathrm{~B}, \mathrm{E}$ & 5,6 & 33549.72 & $5,0,7$ & {$[2,3]$} \\
\hline 33583.38 & $A, B, D$ & 6,7 & 33584.18 & 6 & {$[2,3]$} \\
\hline 33588.94 & $\mathrm{~B}, \mathrm{E}$ & 5,6 & 33589.2 & $5,6,7$ & {$[2]$} \\
\hline 33591.33 & $A, B, D$ & 6,7 & 33590.90 & 6,7 & [3] \\
\hline 33624.54 & $A, B, E$ & 6 & 33624.70 & 6 & \\
\hline 33660.51 & $\mathrm{D}, \mathrm{E}$ & 5,6 & & & \\
\hline 33663.93 & $B, D$ & $5,6,7$ & 33663.72 & $5,6,7$ & {$[2,3]$} \\
\hline 33737.32 & $A, D, E$ & 6 & 33737.46 & 6,7 & {$[2,3]$} \\
\hline 33751.90 & $\mathrm{~A}, \mathrm{E}$ & 6 & 33752.03 & 6 &,$[3]$ \\
\hline 33765.70 & $\mathrm{D}, \mathrm{E}$ & 5,6 & & & \\
\hline 33777.17 & $\mathrm{D}, \mathrm{E}$ & 5,6 & & & \\
\hline 33801.17 & $A, D$ & 6,7 & 33801.00 & $6,7,8$ & [3] \\
\hline 33815.33 & $\mathrm{D}, \mathrm{E}$ & 5,6 & & & \\
\hline 33833.37 & $A, E$ & 6 & 33833.35 & 6 & {$[2,3]$} \\
\hline 33837.85 & $\mathrm{~A}, \mathrm{D}, \mathrm{E}$ & 6 & 33837.82 & 6,7 & {$[2,3]$} \\
\hline 33874.27 & $\mathrm{D}, \mathrm{E}$ & 5,6 & 33874.31 & 5 & {$[2,3]$} \\
\hline 33907.38 & $A, E, E$ & 6 & 33907.76 & 6 & {$[2,3]$} \\
\hline 33940.05 & $A, D$ & 6,7 & 33939.76 & $6^{\circ}$ & [ 3] \\
\hline
\end{tabular}

\footnotetext{
$A=16900.38 \mathrm{~cm}^{-1} ; J=7 . B=16505.77 \mathrm{~cm}^{-1} ; J=6$.

$\mathrm{D}=17361.90 \mathrm{~cm}^{-1} ; J=6 . \mathrm{E}=17369.56 \mathrm{~cm}^{-1} ; J=5$.

$\mathrm{C}=16929.76 \mathrm{~cm}^{-1} ; J=5$.
}

$\left({ }^{5} \mathrm{~K}_{6} \leftarrow{ }^{5} \mathrm{~L}_{6}^{0}\right)$ ，扫描 $\mathrm{DL}_{2}$ 测得的双色三光子电离谱的部分谱图．在图 1 中 $\mathrm{s}$ 表示单色峰，其余 为双色峰。对另外四个铀原子第一步跃迁，591.54nm $\left({ }^{7} \mathrm{M}_{7} \leftarrow{ }^{5} \mathrm{~L}_{6}^{0}\right) ; 590.51 \mathrm{~nm}\left({ }^{7} \mathrm{~K}_{5} \leftarrow{ }^{5} \mathrm{~L}_{6}^{0}\right)$; $575.81 \mathrm{~nm}\left({ }^{7} \mathrm{~L}_{6} \leftarrow{ }^{5} \mathrm{~L}_{6}^{0}\right)$ 和 $575.56 \mathrm{~nm}\left({ }^{5} \mathrm{I}_{5} \leftarrow{ }^{5} \mathrm{~L}_{6}^{0}\right), \mathrm{DL}_{2}$ 分别进行扫描获得另外四张双色三光子 电离谱图. 每一个谱图均重复三次。在本工作中,共采用了五个跃迁作为第一步激发,它们的 


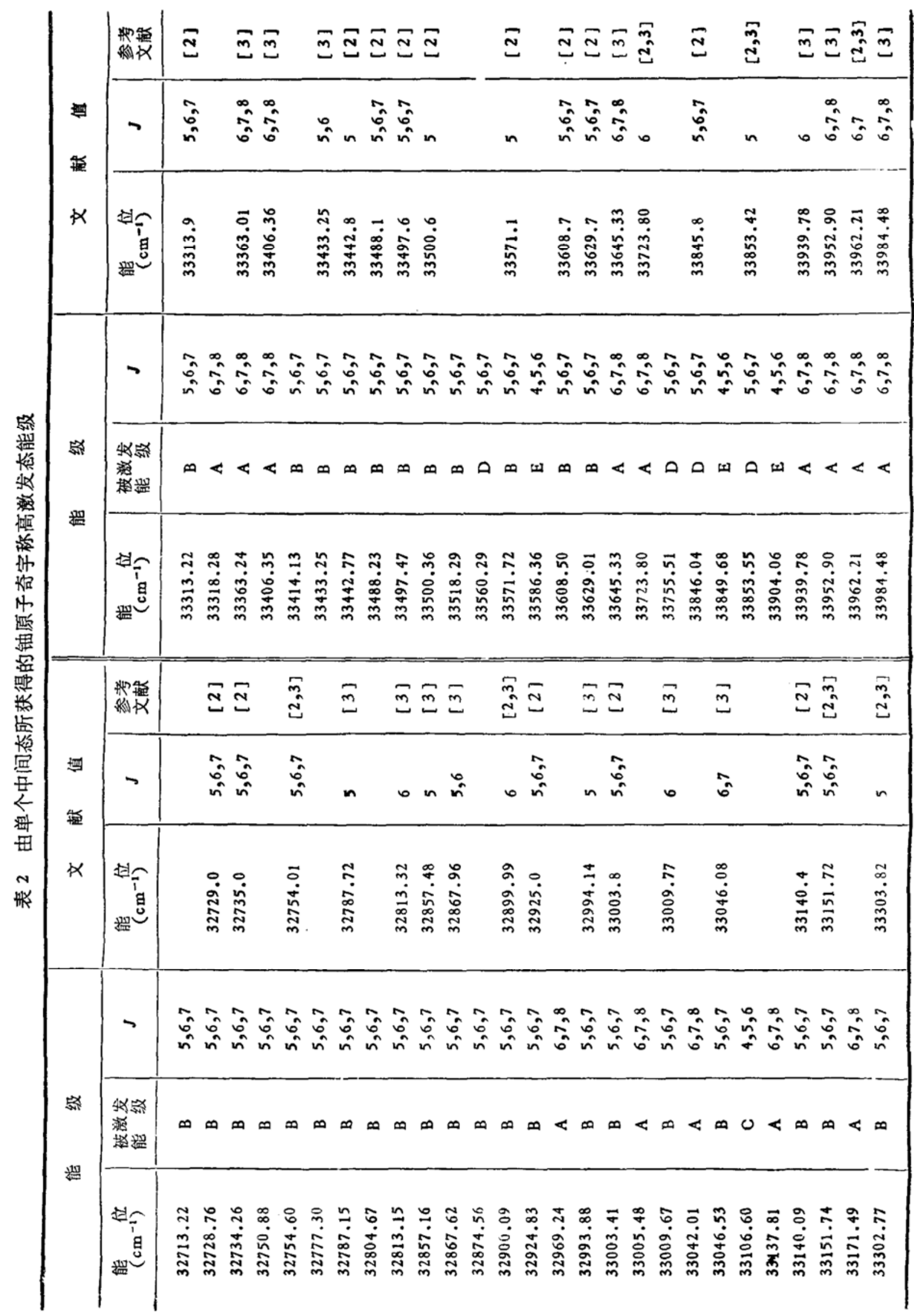




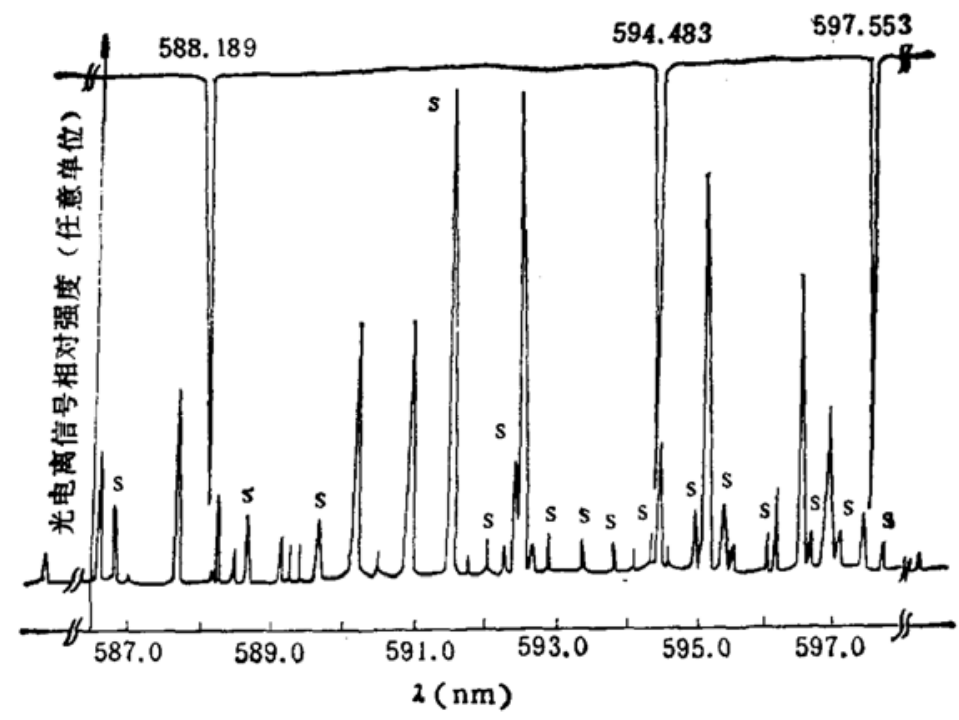

图 1

$J$ 值分别是 7,6 或 5. 我们希望观察到由这三个不同 $J$ 值的第一激发态能级跃迁到同一个高 激发态能级的情况. 那么, 根据 $J$ 值选择定则, 就可确定高激发态能级的 $J$ 值. 在表 1 中给出 了 36 个能级, 其中新确定 $J$ 值的有 10 个. 在表 2 中给出了 54 个能级, 这些能级的 $J$ 值具有 \pm 1 的不确定性.

\section{参考文献}

[ 1 ] Palmer, B. A., Keler, R. A., Engleman, R. Jr., L.os Alamos Scientific Laboratory, Report LA-8251-MS (1980).

[2] Carlson, L. R., Paisner, J. A., Worden, E. F. et al., J. Opt. Soc. Am., 66(1976), 846-853.

[ 3 ] Miron, E., David, R., Erez, G. et al., J. Opt. Soc. Am., 69(1979), 256-264.

[4] Jin Changtai, Liang Xiuqing, Wang Chengfei et al., Chinese Science Bulletin, 37(1992), 1: $30-33$. 